\title{
Minimal linear codes from Hermitian varieties and quadrics
}

\author{
Matteo Bonini ${ }^{1} \cdot$ Stefano Lia $^{2} \cdot$ Marco Timpanella $^{3}$
}

Received: 10 November 2020 / Accepted: 30 January 2021 / Published online: 24 February 2021

(c) The Author(s) 2021

\begin{abstract}
In this note we investigate minimal linear codes arising from Hermitian varieties and quadrics. We study their parameters and formulate some open problems about their weight distribution.
\end{abstract}

Keywords Minimal codes $\cdot$ Linear codes $\cdot$ Secret sharing schemes $\cdot$ Hermitian varieties · Quadrics

Mathematics Subject Classification 94B05 · 94A62 - 51E21

\section{Introduction}

Let $\mathbb{F}_{q}$ be the finite field with $q$ elements, and let $C$ be a $k$-dimensional vectorial subspace of $\mathbb{F}_{q}^{n}$, where $n \in \mathbb{N}$. From now on, we will call $C$ a $[n, k]$ linear code endowed with the Hamming metric, i.e. the distance between two codewords (elements of $C$ ) is given by the number of the coordinate positions in which the two elements differ.

For a codeword $c \in C$, the support of $c$, denoted by $\operatorname{Supp}(\mathrm{c})$, is the set of its nonzero coordinate positions, and the weight of $c$ is $\operatorname{wt}(c)=\# \operatorname{Supp}(\mathrm{c})$. A codeword $c$ is said to be minimal if its support determines $c$ up to a scalar multiplication, i.e. if $c^{\prime} \in C$ is linear independent with $c$, then $\operatorname{Supp}\left(\mathrm{c}^{\prime}\right) \nsubseteq \operatorname{Supp}(\mathrm{c})$. Minimal codewords were employed by Massey (see $[10,11])$ for the construction of a perfect and ideal

Stefano Lia

stefano.lia@unibas.it

Matteo Bonini

matteo.bonini@ucd.ie

Marco Timpanella

marco.timpanella@unibas.it

1 School of Mathematics and Statistics, University College Dublin, Dublin, Ireland

2 Department of Mathematics, Computer Science and Economics, University of the Basilicata, Potenza, Italy

3 Departement of Mathematics and Physics, University of the Campania, Luigi Vanvitelli, Caserta, Italy 
SSS, in which the access structure is determined by the set of the minimal codewords of a linear code.

Unfortunately, the determination of the set of minimal codewords of a given code is a difficult task. Therefore, obtaining the access structure of Massey's SSS is very challenging. This fact led to the study of codes for which every non-zero codeword is minimal, that are called minimal codes.

A useful tool to construct minimal codes was given by Ashikhmin and Barg (see [2]).

Lemma 1 Let $C$ be a linear code over $\mathbb{F}_{q}$, and denote by $\mathrm{wt}_{\text {min }}$ and $\mathrm{wt}_{\max }$ the minimum and maximum nonzero weights in $\mathcal{C}$, respectively. If $\frac{\mathrm{wt}_{\min }}{\mathrm{wt}_{\max }}>\frac{q-1}{q}$ (called the $\mathrm{AB}$ condition from now on), then $C$ is minimal.

Since the study of minimal codes has attracted much attention in recent years, many families of minimal linear codes satisfying $\mathrm{AB}$ have been constructed, e.g. see $[5]$.

However, AB is only a sufficient condition and, for this reason, a relative new research line consists in finding examples of minimal codes violating AB. Families of these codes were first constructed in [7], whereas [6,8] give the first infinite family of minimal codes for the binary and ternary case, respectively. Afterwards, in [3] the first examples of minimal linear codes for every field of odd characteristic were constructed.

Following the geometrical approach of [3], in [4] it was proved that it is possible to construct families of minimal codes through the study of cutting blocking sets.

Recently, in $[1,13]$ the authors found out independently that cutting blocking sets not only determine minimal codes, but they are actually in bijection with them.

Therefore, minimal codes and algebraic varieties over finite fields are very related objects. In this note we investigate families of minimal codes arising from celebrated objects in finite geometry, i.e. Hermitian varieties and quadrics, giving some information about their weight distribution.

\section{Preliminaries}

\subsection{Blocking sets}

Hereafter, we will denote with $\mathbb{F}_{q}$ the finite field with $q$ elements, where $q=p^{h}$ is a prime power, and with $\operatorname{PG}(n, q)$ the projective space of dimension $n \in \mathbb{N}$ over $\mathbb{F}_{q}$. A subspace of dimension $r$ in $\operatorname{PG}(n, q)$ is denoted by $\Pi_{r}$. For a set of homogeneous polynomials $f_{1}, \ldots, f_{r} \in \mathbb{F}_{q}\left[X_{0}, \ldots, X_{n}\right], V_{p}\left(f_{1}, \ldots, f_{r}\right)$ is the projective variety associated to $f_{1}, \ldots, f_{r}$. With $\Pi_{r} V_{p}\left(f_{1}, \ldots, f_{r}\right)$ we denote a cone in $\operatorname{PG}(n, q)$ having vertex $\Pi_{r}$ and base $V_{p}\left(f_{1}, \ldots, f_{r}\right)$, i.e. $\Pi_{r} V_{p}\left(f_{1}, \ldots, f_{r}\right)$ is the join of $\Pi_{r}$ to $V_{p}\left(f_{1}, \ldots, f_{r}\right)$.

In the following, we recall some useful basic definitions on blocking sets; for an exhaustive reference we point to [12, Chapter 3]. 
Definition 1 A $k$-blocking set is a subset of $\operatorname{PG}(n, q)$ intersecting all $(n-k)$-dimensional subspaces. When $k=1$, it is simply called a blocking set.

The dimension of a $k$-blocking set corresponds to the dimension of the subspace generated by its elements.

A $(k, s)$-blocking set in $\operatorname{PG}(n, q)$, where $n>k$, is a $k$-blocking set that does not contain a $s$-dimensional subspace.

As we already mentioned, in order to construct minimal linear codes as in [4] we need to introduce a particular class of blocking set.

Definition 2 A $k$-blocking set in $\operatorname{PG}(n, q)$ is cutting if its intersection with every $(n-k)$-dimensional subspace is not contained in any other $(n-k)$-dimensional subspace.

\subsection{Minimal codes from cutting blocking sets}

For every homogeneous function $f: \mathbb{F}_{q}^{n+1} \rightarrow \mathbb{F}_{q}$, let $\widetilde{\mathcal{C}}_{f}$ be the linear code defined as

$$
\widetilde{\mathcal{C}}_{f}:=\left\{c_{u, v}=(u f(x)+v \cdot x)_{x \in \mathrm{PG}(n, q)} \mid u \in \mathbb{F}_{q}, v \in \mathbb{F}_{q}^{n+1}\right\} .
$$

Here we write a point of $\operatorname{PG}(n, q)$ in standard notation, i.e. the first nonzero coordinate from the left is 1 . The resulting code does not depend on this choice.

Remark 1 If $f$ is non-linear, then $\widetilde{\mathcal{C}}_{f}$ is a $\left[\left(q^{n+1}-1\right) /(q-1), n+2\right]$ code and a generator matrix is obtained by extending the generator matrix of a simplex code by a line from the evaluation of the homogeneous function $f$.

In particular, for any $v \in\left(\mathbb{F}_{q}^{n+1}\right)^{*}, \operatorname{wt}(c(0, v))=\left(q^{n+1}-q^{n}\right) /(q-1)$, whereas for any $u \in \mathbb{F}_{q}^{*} \operatorname{wt}(c(u, 0))=\left(q^{n+1}-1\right) /(q-1)-\# V_{p}(f)$.

The following result gives a sufficient condition to construct minimal codes (see [4] for details).

Theorem 1 Let $f: \underset{q}{\mathbb{F}_{q}^{n+1}} \rightarrow \mathbb{F}_{q}$ be a homogeneous function such that:

(a) $V_{p}(f)$ is a n-dimensional cutting $(1, n-1)$-blocking set in $\mathrm{PG}(n, q)$,

(b) for every $v \in \mathbb{F}_{q}^{n+1} \backslash\{0\}$, there exists $x \in \mathbb{F}_{q}^{n+1}$ such that $f(x)+v \cdot x=0$ and $x \notin V_{p}(f)$

then $\widetilde{\mathcal{C}}_{f}$ defined as in $(1)$ is a $\left[\left(q^{n+1}-1\right) /(q-1), n+2\right]$ minimal code over $\mathbb{F}_{q}$.

Depending on the variety $V_{p}(f)$, the code $\widetilde{\mathcal{C}}_{f}$ may not satisfy the $\mathrm{AB}$ condition.

Lemma 2 [4, Lemma 4.9] If \# $V_{p}(f) \geq \frac{2 q^{n}-q^{n-1}-1}{q-1}$, then the code $\widetilde{\mathcal{C}}_{f}$ as in (1) does not satisfy the $\mathrm{AB}$ condition. 
Our aim is to show that properties (a) and (b) of Theorem 1 hold in the case of Hermitian varieties and quadrics.

\section{Minimal codes from Hermitian varieties}

Our notations and terminologies are standard, see [9]. Let $f_{r}, r \in\{1, \ldots, n\}$, be a canonical Hermitian form in $\mathbb{F}_{q^{2}}\left[X_{0}, X_{1}, \ldots, X_{n}\right]$, i.e.

$$
f_{r}=X_{0}^{q+1}+\cdots+X_{r}^{q+1} .
$$

The variety $V_{p}\left(f_{r}\right)$ is a Hermitian variety in $\operatorname{PG}\left(n, q^{2}\right)$. If $r=n$, the Hermitian variety $V_{p}\left(f_{n}\right)$ is non-singular and it is denoted by $\mathcal{H}_{n}$.

The following result is a corollary of [9, Lemma 2.20] and gives information about the intersections of $\mathcal{H}_{n}$ with hyperplanes.

Proposition 1 The intersection between $\mathcal{H}_{n}$ and an hyperplane of $\operatorname{PG}\left(n, q^{2}\right)$ is either a non-singular Hermitian variety $\mathcal{H}_{n-1}$ or a cone $\Pi_{0} \mathcal{H}_{n-2}$.

Proposition 2 [9, Lemma 2.18] The dimension of a subspace of maximum dimension lying on $\mathcal{H}_{n}$ equals $\lfloor(n-1) / 2\rfloor$.

Propositions 1 and 2 allow to prove that Theorem 1 can be applied to non-singular Hermitian varieties.

Proposition 3 Condition (a) of Theorem 1 holds for non-singular Hermitian varieties.

Proof We show that $\mathcal{H}_{n}$ is an $n$-dimensional $(1, n-1)$ cutting blocking set of $\operatorname{PG}(n, q)$. First, by Proposition $1, \mathcal{H}_{n}$ is an $n$-dimensional blocking set. Moreover $\mathcal{H}_{n}$ cannot contain a hyperplane (see Proposition 2), and hence it is a $(1, n-1)$ blocking set. Finally, since by Proposition 1 the intersection of $\mathcal{H}_{n}$ with any hyperplane cannot be contained in a space of dimension $n-2$, the claim follows.

Proposition 4 Let $\mathcal{H}_{n}=V_{p}(f)$ be a non-singular Hermitian variety in canonical form. Then Condition (b) of Theorem 1 holds.

Proof Let $v=\left(v_{0}, \ldots, v_{n}\right) \in \mathbb{F}_{q^{2}}^{n+1} \backslash\{0\}$ and let $i \in\{0, \ldots, n\}$ such that $v_{i} \neq 0$. If $x \in \mathbb{F}_{q^{2}}^{n+1}$ is such that $x_{j}=0$ for $j \neq i$ and $x_{i}=-v_{i}^{q}$, then $f(x)+v \cdot x=0$ and $f(x) \stackrel{q^{2}}{=} v_{1}^{q+1} \neq 0$.

By Propositions 3 and 4, Theorem 1 applies to $f=X_{0}^{q+1}+\ldots+X_{n}^{q+1}$ and $\widetilde{\mathcal{C}}_{f}$ is a $\left[\left(q^{2 n}-1\right) /\left(q^{2}-1\right), n+2\right]$ minimal code over $\mathbb{F}_{q^{2}}$. 
Remark 2 Since the number of points of a non-singular projective Hermitian variety of dimension $n$ is

$$
\mathcal{P}\left(\mathcal{H}_{n}\right)=\frac{\left[q^{n+1}+(-1)^{n}\right]\left[q^{n}-(-1)^{n}\right]}{q^{2}-1},
$$

Lemma 2 implies that $\widetilde{\mathcal{C}}_{f}$ does not satisfy the $\mathrm{AB}$ condition.

\section{On the weight distribution of minimal codes from $\mathcal{H}_{n}$}

In this section, $\mathcal{A}\left(\mathcal{H}_{n}\right)=\mathcal{P}\left(\mathcal{H}_{n}\right)-\mathcal{P}\left(\mathcal{H}_{n-1}\right)$ denotes the number of affine points of a non-singular Hermitian variety of $\operatorname{PG}\left(n, q^{2}\right)$ and $\square_{q}^{*}$ is the set of nonzero squares of $\mathbb{F}_{q}$. We collect here the weight of some codewords, as a first approach to the weight distribution problem. The next proposition is from Remark 1.

Proposition 5 For every $v \in \mathbb{F}_{q^{2}}^{n+1}$ and $u \in \mathbb{F}_{q^{2}}$, the weights of $c_{0, v}$ and $c_{u, 0}$ are $\operatorname{wt}\left(c_{0, v}\right)=\frac{q^{2(n+1)}-q^{2 n}}{q^{2}-1}$, and $\operatorname{wt}\left(c_{u, 0}\right)=\frac{q^{2(n+1)}-1}{q^{2}-1}-\mathcal{P}\left(\mathcal{H}_{n}\right)$, respectively.

Proposition 6 For every $u \in \mathbb{F}_{q^{2}}^{*}$ let $v_{u}=(-u, 0, \ldots, 0) \in \mathbb{F}_{q^{2}}^{n+1}$. The weight of $c_{u, v_{u}}$ is

$$
\operatorname{wt}\left(c_{u, v_{u}}\right)=\frac{q^{2(n+1)}-1}{q^{2}-1}-\left(q^{2} \mathcal{P}\left(\mathcal{H}_{n-1}\right)+1\right) .
$$

Proof Let $A_{n}$ be the number of $\left(x_{0}, \ldots, x_{n}\right) \in \mathbb{F}_{q^{2}}^{n+1}$ such that the first non-zero coordinate is 1 and $u\left(x_{0}^{q+1}+\cdots+x_{n}^{q+1}\right)-u x_{0}=0$. Then clearly $\operatorname{wt}\left(c_{u, v_{u}}\right)=\frac{q^{2(n+1)}-1}{q^{2}-1}-A_{n}$. Also, $A_{n}=B_{n}+C_{n}$ where $B_{n}$ is the number of solutions $\left(1, x_{1}, \ldots, x_{n}\right) \in \mathbb{F}_{q^{2}}^{n+1}$ of $x_{1}^{q+1}+\cdots+x_{n}^{q+1}=0$ and $C_{n}$ is the number of solutions $\left(0, x_{1}, \ldots, x_{n}\right) \in \mathbb{F}_{q^{2}}^{q^{2}}$ of $x_{1}^{q+1}+\cdots+x_{n}^{q+1}=0$ such that the first non-zero coordinate equals 1 . It is readily seen that $B_{n}=\left(q^{2}-1\right) \mathcal{P}\left(\mathcal{H}_{n-1}\right)+1$, and $C_{n}=\mathcal{P}\left(\mathcal{H}_{n-1}\right)$, whence the claim follows.

Proposition 7 For every a in $\mathbb{F}_{q^{2}} \backslash \mathbb{F}_{q}$, the weight of $c_{1, a e_{1}}$, where $e_{1}$ is the first vector of the canonical basis of $\mathbb{F}_{q^{2}}^{n+1}$, is

$$
\operatorname{wt}\left(c_{1, a e_{1}}\right)=\frac{q^{2(n+1)}-1}{q^{2}-1}-\mathcal{P}\left(\mathcal{H}_{n-1}\right)
$$

Proof Let $\tilde{A}_{n}$ be the number of solutions of $x_{0}^{q+1}+\cdots+x_{n}^{q+1}+a x_{0}=0$ such that the first nonzero coordinate equals 1 . Then $\operatorname{wt}\left(c_{1, a e_{1}}\right)=\frac{q^{2(n+1)}-1}{q^{2}-1}-\tilde{A}_{n}$ and $\tilde{A}_{n}=\tilde{B}_{n}+\tilde{C}_{n}$, where $\tilde{B}_{n}$ is the number of solutions $\left(1, x_{1}, \ldots, x_{n}\right) \in \mathbb{F}_{q^{2}}^{n+1}$ of $x_{1}^{q+1}+\cdots+x_{n}^{q+1}+a+1=0$ and $\tilde{C}_{n}$ is the number of solutions $\left(0, x_{1}, \ldots, x_{n}\right) \in \mathbb{F}_{q^{2}}^{n+1}$ 
of $x_{1}^{q+1}+\cdots+x_{n}^{q+1}=0$ such that the first non-zero coordinate equals 1 . Now, $a \notin \mathbb{F}_{q}$ yields $\tilde{B}_{n}=0$. On the other hand, $\tilde{C}_{n}=\mathcal{P}\left(\mathcal{H}_{n-1}\right)$ and the claim follows.

Lemma 3 Let $q$ be odd. Then the number of $(n+1)$-tuples $\left(x_{0}, \ldots, x_{n}\right)$ such that $x_{0}^{q+1}+\cdots+x_{n}^{q+1}+x_{i}=0$ and such that the first nonzero coordinate equals 1 and at most the $i$-th coordinate is nonzero, is

$$
\begin{aligned}
E_{i}= & {\left[\left(q^{2}-1\right) \cdot \mathcal{P}\left(\mathcal{H}_{n-i-1}\right)+1\right] \cdot\left(\sum_{j=0}^{i} \tilde{E}_{j}\right)+} \\
& +\left[q \cdot \frac{q^{2 i}-1}{q^{2}-1}+1-\left(\sum_{j=0}^{i} \tilde{E}_{j}\right)\right] \cdot \mathcal{A}\left(\mathcal{H}_{n-i}\right)
\end{aligned}
$$

where $\tilde{E}_{j}$ is given by

$$
\begin{cases}1 \text { if } q \equiv 0 & (\bmod 3) \\ 2 \text { if } q \equiv 1 & (\bmod 3) \\ 0 \text { if } q \equiv 2 & (\bmod 3)\end{cases}
$$

if $j=1$, and by

$$
\left\{\begin{array}{l}
\mathcal{P}\left(\mathcal{H}_{j-2}\right) \cdot\left(q^{2}-1\right)+1+2 \cdot \frac{q-1}{2} \mathcal{A}\left(\mathcal{H}_{j-1}\right) \text { if } q \equiv 0 \quad(\bmod 3) \\
\mathcal{A}\left(\mathcal{H}_{j-1}\right)+2 \cdot q \cdot \mathcal{A}\left(\mathcal{H}_{j-1}\right) \text { if } q \equiv 2 \quad(\bmod 3) \\
(q-2) \mathcal{A}\left(\mathcal{H}_{j-1}\right)+2\left(q^{2}-1\right) \mathcal{P}\left(\mathcal{H}_{j-2}\right)+2 \text { if } q \equiv 1 \quad(\bmod 3)
\end{array}\right.
$$

for $j \geq 2$.

Proof Write $x_{0}^{q+1}+\cdots+x_{n}^{q+1}+x_{i}=X+Y$, where $X=x_{0}^{q+1}+\cdots+x_{i}^{q+1}+x_{i}$ and $Y=x_{i+1}^{q+1}+\cdots+x_{n}^{q+1}$. For an $(n+1)$-tuple satisfying $X+Y=0$, either $X=0$ and $Y=0$ or $X \neq 0$. In both cases $X=-Y \in \mathbb{F}_{q}$. Let $\tilde{E}_{j}, j \geq 1$, be the number of $(j+1)$ -tuples $\left(1, x_{1}, \ldots, x_{j}\right) \in \mathbb{F}_{q^{2}}^{j+1}$ such that $x_{1}^{q+1}+\cdots+x_{j}^{q+1}+x_{j}+1=0$. Then $X=0$ has $\sum_{j=1}^{i} \tilde{E}_{j}$ solutions, while $Y=0$ has $\left(q^{2}-1\right) \cdot \mathcal{P}\left(\mathcal{H}_{n-i-1}\right)+1$ solutions. On the other hand, the number of $(i+1)$-tuple $\left(x_{0}, \ldots, x_{i}\right)$ such that the first non-zero coordinate has value 1 and $X \in \mathbb{F}_{q}$ is $q \cdot \frac{q^{2 i}-1}{q^{2}-1}+1$. Hence the case $X \neq 0$ and $X+Y=0$ occurs in $\left[q \cdot \frac{q^{2 i}-1}{q^{2}-1}+1-\left(\sum_{j=0}^{i} \tilde{E}_{j}\right)\right] \cdot \mathcal{A}\left(\mathcal{H}_{n-i}\right)$ cases.

To compute $\tilde{E}_{j}$ observe that if $x_{1}^{q+1}+\cdots+x_{j}^{q+1}+x_{j}+1=0$, then $x_{j} \in \mathbb{F}_{q}$. If $j=1$ then $x_{1}^{q+1}+\cdots+x_{j}^{q+1}+x_{j}+1=0$ reads $x_{1}^{2}+x_{1}+1=0$, which has one solution if $p=3$, and two solutions if $-3 \in \square_{q}^{*}$, i.e. if $q \equiv 1(\bmod 3)$; zero otherwise. Let $j \geq 2$. Then $x_{1}^{q+1}+\cdots+x_{j}^{q+1}+x_{j}+1=0$ reads $x_{1}^{q+1}+\cdots+x_{j-1}^{q+1}+x_{j}^{2}+x_{j}+1=0$. For any choice of $x_{1}, \ldots, x_{j-1}$, let $c=x_{1}^{q+1}+\cdots+x_{j-1}^{q+1}+1$. If $c=1 / 4$ then the equation $x_{j}^{2}+x_{j}+c=0$ has one solution in $x_{j}$, whereas if $1-4 c \in \square_{q}^{*}$, there are two solutions in $x_{j}$. The value $c=1 / 4$ is reached when 


$$
x_{1}^{q+1}+\cdots+x_{j-1}^{q+1}+1=1 / 4 .
$$

If $p \neq 3$, this happens $\mathcal{A}\left(\mathcal{H}_{j-1}\right)$ times, whereas, if $p=3$ it occurs $\left[\left(q^{2}-1\right) \cdot \mathcal{P}\left(\mathcal{H}_{j-2}\right)+1\right]$ times.

Assume now $1-4 c \in \square_{q}^{*}$. Then there exists $w \in \mathbb{F}_{q}^{*}$ such that

$$
1-4\left(x_{1}^{q+1}+\cdots+x_{j-1}^{q+1}+1\right)=w^{2} .
$$

If $w^{2}=-3$, (4) reads $x_{1}^{q+1}+\cdots+x_{j-1}^{q+1}=0$, which yields $\left(q^{2}-1\right) \cdot \mathcal{P}\left(\mathcal{H}_{j-2}\right)+1$ choices for $\left(x_{1}, \ldots, x_{j-1}\right)$. In the other case, that is $w^{2} \neq-3$, there are $\mathcal{A}\left(\mathcal{H}_{j-1}\right)$ choices.

Since there are $\frac{q-1}{2}$ nonzero squares in $\mathbb{F}_{q}$ and $-3 \notin \square_{q}^{*}$ if and only if $q \equiv 2(\bmod 3)$, the claim follows.

Proposition 8 Let qbe odd. Then the weight of $c_{1, e_{i}}$, where $e_{i}$ is the $i$-th vector of the standard basis of $\underset{q^{2}}{\mathbb{F}^{n+1}}$, is

$$
\operatorname{wt}\left(c_{1, e_{i}}\right)=\frac{q^{2(n+1)}-1}{q^{2}-1}-\left(E_{i}+\mathcal{P}\left(\mathcal{H}_{n-i-1}\right)\right)
$$

where $E_{i}$ is given by (3).

Proof Since the number of solutions of the equation $x_{0}^{q+1}+\cdots+x_{i}^{q+1}+x_{i}+1+x_{i+1}^{q+1}+\cdots+x_{n}^{q+1}=0$ such that their first non-zero component is at least the $(i+1)$-th one is $\mathcal{P}\left(\mathcal{H}_{n-i-1}\right)$, the claim follows from Lemma 3.

\section{Minimal codes from quadrics}

For a quadratic form $f \in \mathbb{F}_{q}\left[X_{0}, \ldots, X_{n}\right]$, with $f=\sum_{i=0}^{n} a_{i} X_{i}^{2}+\sum_{i<j} a_{i, j} X_{i} X_{j}$, the associated variety $V_{p}(f)$ is a quadric in $\operatorname{PG}(n, q)$. If $f$ is non-degenerate, i.e. $f$ cannot be reduced by a linear transformation to a quadratic form in less variables, $V_{p}(f)$ is a non-singular quadric and it is denoted by $\mathcal{Q}_{n}$. Up to projective equivalence there are one or two distinct non-singular quadrics according to $n$ being even or odd; see [9, Chapter 1].

\section{Proposition 9}

- If $n$ is even a non-singular quadric $\mathcal{Q}_{n}$ is projectively equivalent to a non-singular quadric $\mathcal{P}_{n}$, called parabolic, where 


$$
\mathcal{P}_{n}=V_{p}\left(X_{0}^{2}+X_{1} X_{2}+\cdots+X_{n-1} X_{n}\right)
$$

- If $n$ is odd a non-singular quadric $\mathcal{Q}_{n}$ is either projectively equivalent to a nonsingular quadric $\mathcal{E}_{n}$, called elliptic, or to a non-singular quadric $\mathcal{H}_{n}$, called hyperbolic, where

$$
\mathcal{E}_{n}=V_{p}\left(g\left(X_{0}, X_{1}\right)+X_{2} X_{3}+\cdots+X_{n-1} X_{n}\right)
$$

with $g\left(X_{0}, X_{1}\right)=d X_{0}^{2}+X_{0} X_{1}+X_{1}^{2}$, irreducible over $\mathbb{F}_{q}, d \in \mathbb{F}_{q}^{*}$, and

$$
\mathcal{H}_{n}=V_{p}\left(X_{0} X_{1}+X_{2} X_{3}+\cdots+X_{n-1} X_{n}\right) .
$$

The quadrics $\mathcal{P}_{n}, \mathcal{E}_{n}$ and $\mathcal{H}_{n}$ as in Proposition 9 are said to be in canonical forms. We say that two non-singular quadrics have the same character if they are both parabolic (character 1), both hyperbolic (character 2) or both elliptic (character 0 ).

Proposition 10 Let $n \geq 3$. The intersection of a non-singular quadric $\mathcal{Q}_{n}$ with an hyperplane is either a non-singular quadric $\mathcal{Q}_{n-1}$ of $\mathrm{PG}(n-1, q)$ or a cone $\Pi_{0} \mathcal{Q}_{n-2}$, where $\mathcal{Q}_{n}$ and $\mathcal{Q}_{n-2}$ have the same character.

Proposition 11 The maximum dimension of a subspace on a non-singular quadric $\mathcal{Q}_{n}$ is either $\frac{1}{2}(n-2), \frac{1}{2}(n-1)$, or $\frac{1}{2}(n-3)$ according to $\mathcal{Q}_{n}$ being respectively parabolic, hyperbolic or elliptic.

This results allow us to prove properties (a) and (b) of Theorem 1 hold for nonsingular quadrics in canonical form.

Proposition 12 Let $n \geq 3$.Then condition (a) of Theorem 1 holds for non-singular quadrics of $\mathrm{PG}(n, q)$.

Proof By Proposition 10, a non-singular quadric is a blocking set of PG $(n, q)$. Moreover, by Proposition $11, \mathcal{Q}_{n}$ cannot contain an hyperplane, whence it is a $(1, n-1)$ -blocking set. Finally, since the intersection of $\mathcal{Q}_{n}$ with an hyperplane cannot be contained in a space of dimension $n-2$ (see Proposition 10), the blocking set is also cutting.

Proposition 13 Let $\mathcal{Q}_{n}=V_{p}(f)$ be a non-singular quadric in canonical form. Then Condition (b) of Theorem 1 holds.

Proof Let $v=\left(v_{0}, \ldots, v_{n}\right) \in \mathbb{F}_{q}^{n+1} \backslash\{0\}$. We prove the claim separately for elliptic, parabolic and hyperbolic quadrics.

(1) If the quadric is $_{n}$ elliptic then $n \geq 3$ and (up to projectivities) $f(x)+v \cdot x$ reads $g\left(x_{0}, x_{1}\right)+\sum_{i=1}^{\frac{n-1}{2}} x_{2 i} x_{2 i+1}+\sum_{i=0}^{n} v_{i} x_{i}$, where $g\left(X_{0}, X_{1}\right)=d X_{0}^{2}+X_{0} X_{1}+X_{1}^{2}$ is an irreducible polynomial and $d \in \mathbb{F}_{q}^{*}$. First, assume $v_{i}=0$ for every $i=2, \ldots, n$. 
Then either $v_{0} \neq 0$ or $v_{1} \neq 0$. Assuming without loss of generalities $v_{0} \neq 0$, then $\bar{x}=\left(1,0,1,-v_{0}-g(1,0), 0, \ldots, 0\right)$ satisfies $f(\bar{x})=-v_{0} \neq 0$ and $f(\bar{x})+v \cdot \bar{x}=0$. Assume now there exists $i \in\{2, \ldots, n\}$ such that $v_{i} \neq 0$. Then, $\bar{x}$ such that

$$
\bar{x}_{j}= \begin{cases}1 & \text { if } j=1, \\ -\frac{v_{1}+g(0,1)}{v_{i}} & \text { if } j=i \\ 0 & \text { otherwise }\end{cases}
$$

satisfies $f(\bar{x})=g(0,1)=1 \neq 0$ and $f(\bar{x})+v \cdot \bar{x}=0$.

(2) If the quadric is parabolic then $f(x)+v \cdot x$ reads $x_{0}^{2}+\sum_{i=1}^{\frac{n}{2}} x_{2 i-1} x_{2 i}+\sum_{i=0}^{n} v_{i} x_{i}$. First, assume $v_{i}=0$ for every $i=1, \ldots, n$. Then $v_{0} \neq 0$ and $\bar{x}=\left(-v_{0}, 0, \ldots, 0\right)$ satisfies $f(\bar{x}) \neq 0$ and $f(\bar{x})+v \cdot \bar{x}=0$. Assume now there exists $i \in\{1 \ldots, n\}$ such that $v_{i} \neq 0$. In this case if $\bar{x}$ is defined by $\bar{x}_{0}=1, \bar{x}_{i}=-\frac{1}{v_{i}}$ and $\bar{x}_{j}=0$ for $j \neq 0, i$, then $\bar{x}$ satisfies $f(\bar{x}) \neq 0$ and $f(\bar{x})+v \cdot \bar{x}=0$.

(3) If the quadric is hyperbolic then $f(x)+v \cdot x$ reads $\sum_{i=0}^{\frac{n-1}{2}} x_{2 i} x_{2 i+1}+\sum_{i=0}^{n} v_{i} x_{i}$. Assume $v_{i} \neq 0, i \in\{0, \ldots, n\}$, and let $\bar{j} \in\left\{0, \ldots, \frac{n-1}{2}\right\}$ such that $2 \bar{j}, 2 \bar{j}+1 \neq i$. Then $\bar{x}$ such that

$$
\bar{x}_{j}= \begin{cases}1 & \text { if } j=\overline{2 j} \text { or } j=2 \bar{j}+1, \\ -\frac{v_{2 \bar{j}}+v_{2 \bar{j}+1}+1}{v_{i}}, & \text { if } j=i, \\ 0, & \text { otherwise }\end{cases}
$$

satisfies $f(\bar{x})=1 \neq 0$ and $f(\bar{x})+v \cdot \bar{x}=0$.

By Propositions 12 and 13, Theorem 1 applies and the codes $\widetilde{\mathcal{C}}_{f}$ arising from nonsingular quadrics in canonical form are $\left[\left(q^{n}-1\right) /(q-1), n+2\right]$ minimal codes over $\mathbb{F}_{q}$. Note that in this case Lemma 2 does not apply, and indeed MAGMA evidences suggest that the $\mathrm{AB}$ condition holds.

\section{Conclusion and open problems}

In this paper we proved that Hermitian varieties and quadrics are cutting blocking sets, and hence they give place to minimal codes. It is worth noting that Theorem 1 and the construction provided in [1] yield two different families of minimal codes. However, while for the codes $\widetilde{\mathcal{C}}_{f}$ Lemma 2 allows to argue about the $\mathrm{AB}$ condition, for the minimal codes arising from [1] this is not possible. In the following we list some open problems:

1. Determine the full weight distribution of the minimal codes $\widetilde{\mathcal{C}}_{f}$ presented in this paper;

2. Study whether the minimal codes arising from Hermitian varieties and quadrics with the construction in [1] do not respect the $\mathrm{AB}$ condition. 
Acknowledgements The research of S. Lia and M. Timpanella was supported by the Italian National Group for Algebraic and Geometric Structures and their Applications (GNSAGA - INdAM). The research of M. Bonini was supported by the Irish Research Council, Grant No. GOIPD/2020/597.

Funding Open access funding provided by Università degli Studi della Basilicata within the CRUICARE Agreement.

Open Access This article is licensed under a Creative Commons Attribution 4.0 International License, which permits use, sharing, adaptation, distribution and reproduction in any medium or format, as long as you give appropriate credit to the original author(s) and the source, provide a link to the Creative Commons licence, and indicate if changes were made. The images or other third party material in this article are included in the article's Creative Commons licence, unless indicated otherwise in a credit line to the material. If material is not included in the article's Creative Commons licence and your intended use is not permitted by statutory regulation or exceeds the permitted use, you will need to obtain permission directly from the copyright holder. To view a copy of this licence, visit http://creativecommons.org/licen ses/by/4.0/.

\section{References}

1. Alfarano, G.N., Borello, M., Neri, A.: A geometric characterization of minimal codes and their asymptotic performance. Adv. Math. Commun. (2020). https://doi.org/10.3934/amc.2020104

2. Ashikhmin, A., Barg, A.: Minimal vectors in linear codes. IEEE Trans. Inf. Theory 44(5), 20102017 (1998)

3. Bartoli, D., Bonini, M.: Minimal linear codes in odd characteristic. IEEE Trans. Inform. Theory 65(7), 4152-4155 (2019)

4. Bonini, M., Borello, M.: Minimal linear codes arising from blocking sets. J. Algebra Comb. (2020). https://doi.org/10.1007/s10801-019-00930-6

5. Carlet, C., Ding, C., Yuan, J.: Linear codes from highly nonlinear functions and their secret sharing schemes. IEEE Trans. Inf. Theory 51(6), 2089-2102 (2005)

6. Chang, S., Hyun, J.Y.: Linear codes from simplicial complexes. Des. Codes Cryptogr. 86(10), 2167-2181 (2018)

7. Cohen, G.D., Mesnager, S., Patey, A.: On minimal and quasi-minimal linear codes, in IMACC 2013: Cryptography and Coding, pp. 85-98. Heidelberg, Germany (2013)

8. Heng, Z., Ding, C., Zhou, Z.: Minimal Linear Codes over Finite Fields. Finite Fields Appl. 54, 176196 (2018)

9. Hirschfeld, J.W.P., Thas, J.A.: General Galois Geometries. Clarendon Press, Oxford, England (2016)

10. J. L. Massey, Minimal codewords and secret sharing, in Proc. 6th Joint Swedish-Russian Int. Workshop on Info. Theory, Mölle, Sweden, Aug., 276-279 (1993)

11. Massey, J.L.: Some applications of coding theory in cryptography, in Codes and Cyphers: Cryptography and Coding IV, Esses. England 33-47, (1995)

12. Storme, L., De Beule, J.: Current Research Topics in Galois Geometry. Nova publishers incorporated, New york (2014)

13. C. Tang, Y. Qiu, Q. Liao, Z. Zhou, Full Characterization of Minimal Linear Codes as Cutting Blocking Sets, avaible online at: arXiv:1911.09867, (2020)

Publisher's Note Springer Nature remains neutral with regard to jurisdictional claims in published maps and institutional affiliations. 